INTERNATIONAL DESIGN CONFERENCE - DESIGN 2018

https://doi.org/10.21278/idc.2018.0162

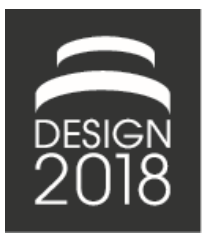

\title{
ASSURANCE OF THE SYSTEM RELIABILITY OF A GEARBOX CONSIDERING PRIOR KNOWLEDGE
}

\author{
M. Bartholdt, A. Grundler, M. Bollmann and B. Bertsche
}

\begin{abstract}
System reliability is predicted for the EcoLife 6-gear automatic transmission from ZF Friedrichshafen AG taking various calculations and testing procedures during product development into account at different system levels. The system reliability predicted in this way is compared to the field reliability derived from the actually observed failure behaviour. The combination of methods as developed, applied and validated in this paper aids in streamlining reliability efforts due to the fact that an updateable process of determining reliabilities at various system levels is formed.
\end{abstract}

Keywords: design evaluation, design support system, design analysis, system reliability, prior knowledge

\section{Motivation and introduction}

The assurance of a newly designed gearbox's reliability is typically achieved during product development by means of calculation and testing. Calculations are typically executed for components of the gearbox and enable technical designers to assure structural durability under operational stresses, or endurance strength. Typically, tests are conducted at different system levels, e.g. at a component, module or system level. Furthermore, they can have different characteristics and purposes, e.g. determining the unit's lifetime through "end-of-life tests" (EoL) or exclusively assuring a certain minimum reliability level for which "success-run tests" (SR) may be implemented.

The two central questions are:

- How can different sources of information be used and combined in order to efficiently generate an assured statement on the overall system reliability?

- Can the reliability levels predicted in this way during product development be confirmed by actually observed values in the field?

\section{General approach}

Prior knowledge gained from calculations, simulations and tests is considered when deriving a statement on the current reliability at the level of a component, subsystem or system. The combination of different items of prior knowledge within a unit (component, module, etc.) is implemented by means of a Bayes'theorem-based approach (Bayes and Price, 1763) based on the Beta distribution as described in Krolo (2004, pp. 39-42) and Krolo and Bertsche (2003). The approximation using the (Beta) distribution moments (method of moments, Thompson and Haynes, 1980) is applied to make use of reliability values for different components to prior knowledge at a higher system level, as described in Maisch (2007, pp. 95-96) or Martz et al. (1988) for example.

The reliability model, i.e. the reliability-relevant system structure with all its subsystems and components to be considered in terms of their reliability, represents the point of departure. So-called "prior probability" 
(a reliability distribution) represents an indispensable input to Bayes' theorem. Bayes' theorem connects this prior probability with additional information from further tests to the posterior probability (another reliability distribution). Validation of the approach is implemented through a comparison of the predicted, theoretical reliability and the actually observed reliability in the field at different system levels.

The adapted approach of applying Bayes' theorem and the method of moments to the EcoLife gearbox is described in more detail in Chapters 3 and 4 respectively.

\section{Combining prior knowledge with current test results}

Prior knowledge gained through calculations, simulations or preceding tests of similar products in terms of their design or of the same product - potentially from a previous generation - is considered in addition to current test results when assessing the reliability of the product. The confidence level $(\approx$ degree of assurance) can thereby be increased and/or the number of test samples required to conduct the corresponding assurance tests reduced.

Bayes' theorem combines prior knowledge in the form of a prior density function with the failure behaviour of the product in its current state to the posterior density function. The latter represents an updated and more accurate estimation of the actual reliability value.

In the course of this paper, the prior density function stems from calculations; the additional information stems from tests.

The posterior density function resulting from Bayes' theorem can be mathematically stated as follows, cf. (Bertsche and Lechner, 2004, p. 274), for example:

$$
f\left(R \mid E_{X}\right)=\frac{P\left(E_{x} \mid R\right) f(R)}{\int_{0}^{1} P\left(E_{x} \mid R\right) f(R) d R}
$$

Here, $\mathrm{f}(\mathrm{R}) \hat{=}$ prior density function, $\mathrm{P}\left(\mathrm{E}_{\mathrm{x}} \mid \mathrm{R}\right) \hat{=}$ conditional probability, $\mathrm{f}\left(\mathrm{R} \mid \mathrm{E}_{\mathrm{x}}\right) \hat{=}$ posterior density function, $\mathrm{R} \triangleq$ reliability.

The conditional probability is typically described as a binominal distribution (Krolo and Bertsche, 2003; Krolo, 2004, p. 37):

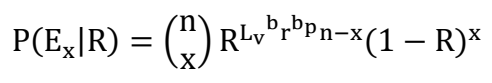

Here, $\mathrm{n} \hat{=}$ sample size, $\mathrm{x} \hat{=}$ number of failures, $\mathrm{b} \hat{=}$ Weibull shape parameter under field conditions, $\mathrm{b}_{\mathrm{p}} \bumpeq$ Weibull shape parameter under test conditions, $\mathrm{L}_{\mathrm{v}}=$ lifetime ratio, $\mathrm{r}=$ acceleration factor.

The conditional probability is described as a binominal distribution (cf. Equation 2) and the prior density function of reliability as a Beta distribution (cf. Equation 3). The Beta distribution's descriptive parameters are $\mathrm{A}_{0}$ and $\mathrm{B}_{0}$, where " 0 " simply denotes the fact that prior knowledge is concerned. The method as described and applied here transfers knowledge stemming from component tests to its full extent. A partial transfer is possible, as is described in (Krolo, 2004, p. 75), for example.

The prior density function of the form

$$
f(R)=\frac{1}{\beta\left(A_{0}, B_{0}\right)} R^{A_{0}-1}(1-R)^{B_{0}-1}
$$

is integrated into the posterior density function by means of Bayes' theorem as follows:

$$
f\left(R \mid E_{X}\right)=\frac{R^{A_{0}-1+n-x}(1-R)^{B_{0}-1+x}}{\beta\left(A_{0}+n-x, B_{0}+x\right)} .
$$

While $\beta(a, b)=\int_{0}^{1} t^{a-1}(1-t)^{b-1} d t$ being the beta function.

This corresponds to a Beta distribution with the descriptive parameters $\mathrm{A}$ and $\mathrm{B}$ :

$$
\mathrm{A}=\left(\mathrm{A}_{0}+\mathrm{n}-\mathrm{x}\right) ; \mathrm{B}=\left(\mathrm{B}_{0}+\mathrm{x}\right)
$$

The mathematical integration of Equation 4 yields the confidence level $\mathrm{P}_{\mathrm{A}}$.

Items of prior knowledge from calculation result in a theoretical reduction of the test sample size $\Delta \mathrm{n}_{\text {cal }}$. This is calculated under the assumption that a step function with the increment at $R\left(t_{s}\right)$ describes $f(R)$ and that a binominal relation exists between reliability and confidence when success runs are the subject matter: 


$$
\Delta \mathrm{n}_{\text {cal }}=\frac{\ln \left(\frac{\mathrm{R}_{\text {target }}\left(\mathrm{t}_{\mathrm{s}}\right)^{2}}{\mathrm{R}_{\text {cal }}\left(\mathrm{t}_{\mathrm{s}}\right)}\right)}{\ln \left(\mathrm{R}_{\text {target }}\left(\mathrm{t}_{\mathrm{s}}\right)\right)}
$$

Here, $R_{\text {target }}\left(t_{s}\right) \hat{=}$ the targeted reliability level at the specified lifetime $t_{s}, R_{\text {cal }}\left(t_{s}\right) \hat{=}$ the calculated reliability level at the specified lifetime $t_{\mathrm{s}}$.

Moreover, it is assumed that $\mathrm{R}_{\text {cal }}$ is linked to a confidence level of $\mathrm{P}_{\mathrm{A}}=50 \%$. The means of calculating $\Delta \mathrm{n}_{\text {cal }}$ replaces the one presented in Krolo (2004, p. 69-70), which is a numerical calculation. The adapted approach presented here is still in line with Dr. Krolo. The underlying step function as the prior knowledge density is illustrated in Figure 1. Its increment is at $R_{\text {target }}\left(t_{s}\right)$. The calculated reliability at the specified lifetime $t_{s}\left(R_{c a l}\left(t_{s}\right)\right)$ needs to be greater than the squared target reliability at the same time $\left(R_{\text {target }}\left(t_{s}\right)\right)$. Otherwise a negative sample size reduction is calculated. In practice, it can typically be assumed that $R_{\text {cal }}>R_{\text {target. }}$ If the calculated reliability was lower than the target reliability, redesigns would be implemented to assure the product's durability through calculations before the test phases began.

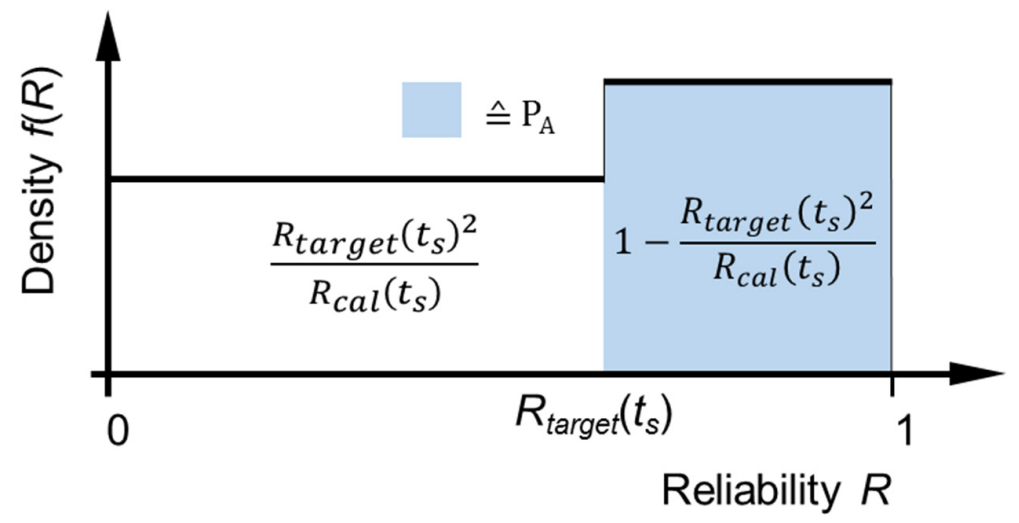

Figure 1. Reliability density function for prior knowledge from calculation

When prior knowledge from calculations is used and when current test results stem from i success-run (SR) or end-of-life (EoL) tests with lifetime ratios $\left(\mathrm{L}_{\mathrm{v}}\right)$, acceleration factors $(\mathrm{r})$ and $\mathrm{x}$ failures (in case of EoL), Equation 5 becomes Equation 7. Here, no prior knowledge from preceding tests is included, which is why $\mathrm{A}_{0}$ and $\mathrm{B}_{0}$ are excluded.

$$
A=\sum_{i}\left(L_{v_{i}} \cdot r_{i}\right)^{b_{i}} \cdot n_{i}+\Delta n_{\text {cal }}-x ; B=1+x
$$

These are the parameters describing the posterior Beta distribution of the reliability density function. The distribution obtained in this manner is used in this paper to determine the reliability value corresponding to a given confidence level.

\section{Combining prior knowledge with higher system levels}

Prior knowledge of a subordinate system level is combined with the reliability density function of a superordinate system level, cf. Figure 2. In this paper, this is achieved through the approximation of the Beta distributions' moments. The prior density function representing a now higher system level can be considered as prior knowledge as described in Chapter 3, and can be combined with current test results at that same system level.

The Beta-distributed parameters of the reliability density function at the aforementioned superordinate system level are calculated by means of Equations 8. This is an approximate approach. The resulting distribution represents the prior density function in case current test results from tests at this system level are available and both pieces of information are to be combined. This is implemented as suggested in (Martz et al., 1988; Maisch, 2007), for example. The expected value $E_{S}(R)$ represents the system distribution's first moment here, cf. Equation 9. The method as described and applied here transfers the 
prior knowledge to its full extent. A partial transfer is possible, as proposed and described in (Martz et al., 1988; Maisch, 2007, p. 96).

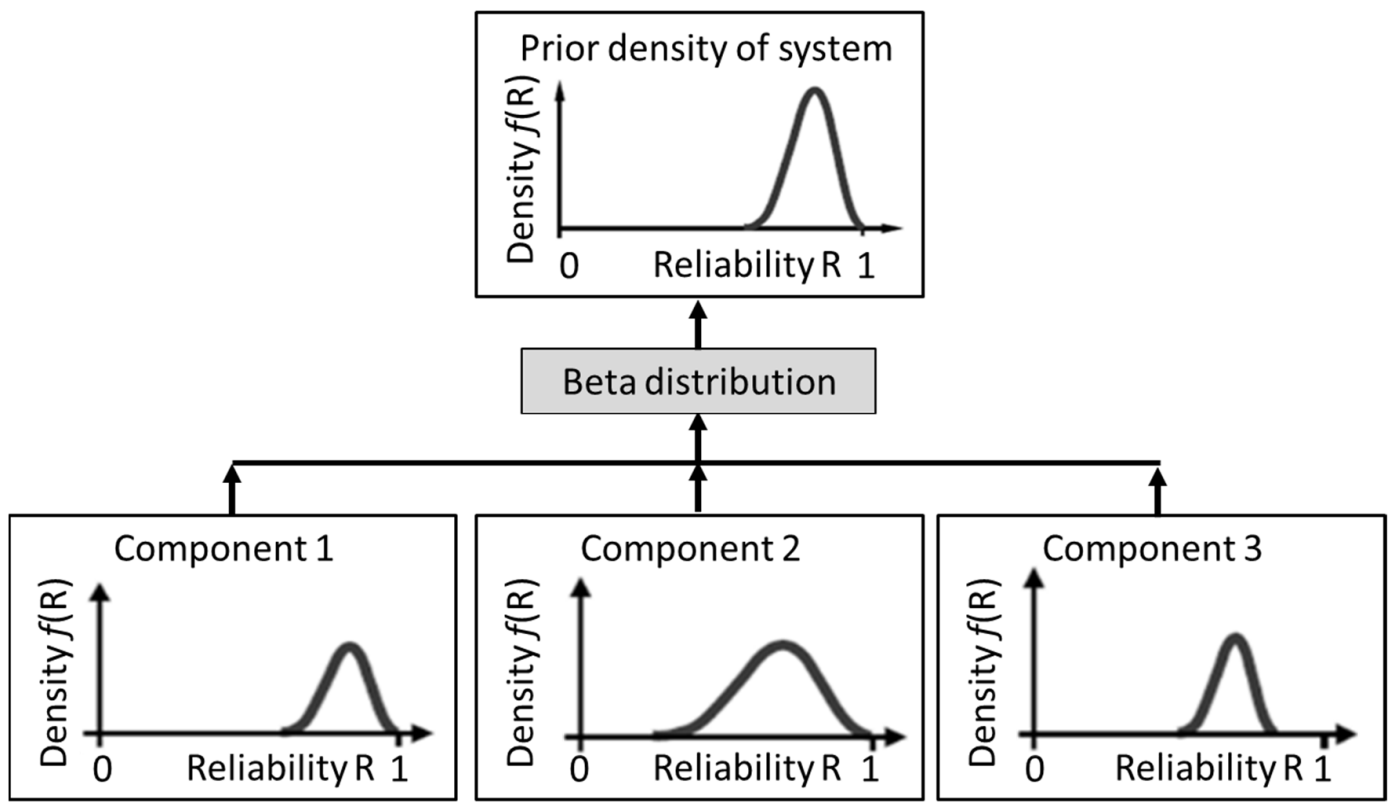

Figure 2. Combination of reliability densities from the component level with the prior density function at a system level (cf. Maisch, 2007, p. 93)

$$
A=(1-E(R)) \cdot \frac{E(R)^{2}}{\operatorname{Var}(R)}-E(R) ; \quad B=A \cdot \frac{1-E(R)}{E(R)}
$$

Here, the expected value of the system's prior density function based on $A_{i}$ and $B_{i}$ of the $\mathrm{j}$ components' Beta distribution:

$$
E_{S}(R)=\prod_{i=1}^{j} \frac{A_{i}}{A_{i}+B_{i}}
$$

The system distribution's variance $\operatorname{Var}_{S}(R)$ is calculated as follows:

$$
\operatorname{Var}_{S}(R)=\prod_{i=1}^{j} \frac{A_{i} \cdot\left(A_{i}+1\right)}{\left(A_{i}+B_{i}\right) \cdot\left(A_{i}+B_{i}+1\right)}-E_{s}^{2}(R)
$$

Further methods exist for determining the system's (prior) density function via the contributing components' reliability distributions. The Mellin transformation can be an exact method under certain circumstances. One other alternative to the method described above is the approximative determination by means of the Monte Carlo simulation. A comparison of the methods is undertaken in Zeiler and Bertsche (2015).

\section{Merged approach}

Figure 3 shows the scheme of the applied approach of combining prior knowledge within a component (cf. Chapter 3) as well as combining the posterior density functions determined in this manner with the superordinate system level (cf. Chapter 4), whereby the obtained density function can be considered a prior density function, which can in turn be combined with test results conducted at that system level.

The Beta distribution stemming from the combination of prior knowledge with the superordinate system level represents the prior density function of this very system level. It is updated with the information from current tests by means of the approach presented in Chapter 3 (Bayes' theorem). The resulting posterior density function at the system level is therefore updated with the information form the corresponding tests, cf. Figure 3. 


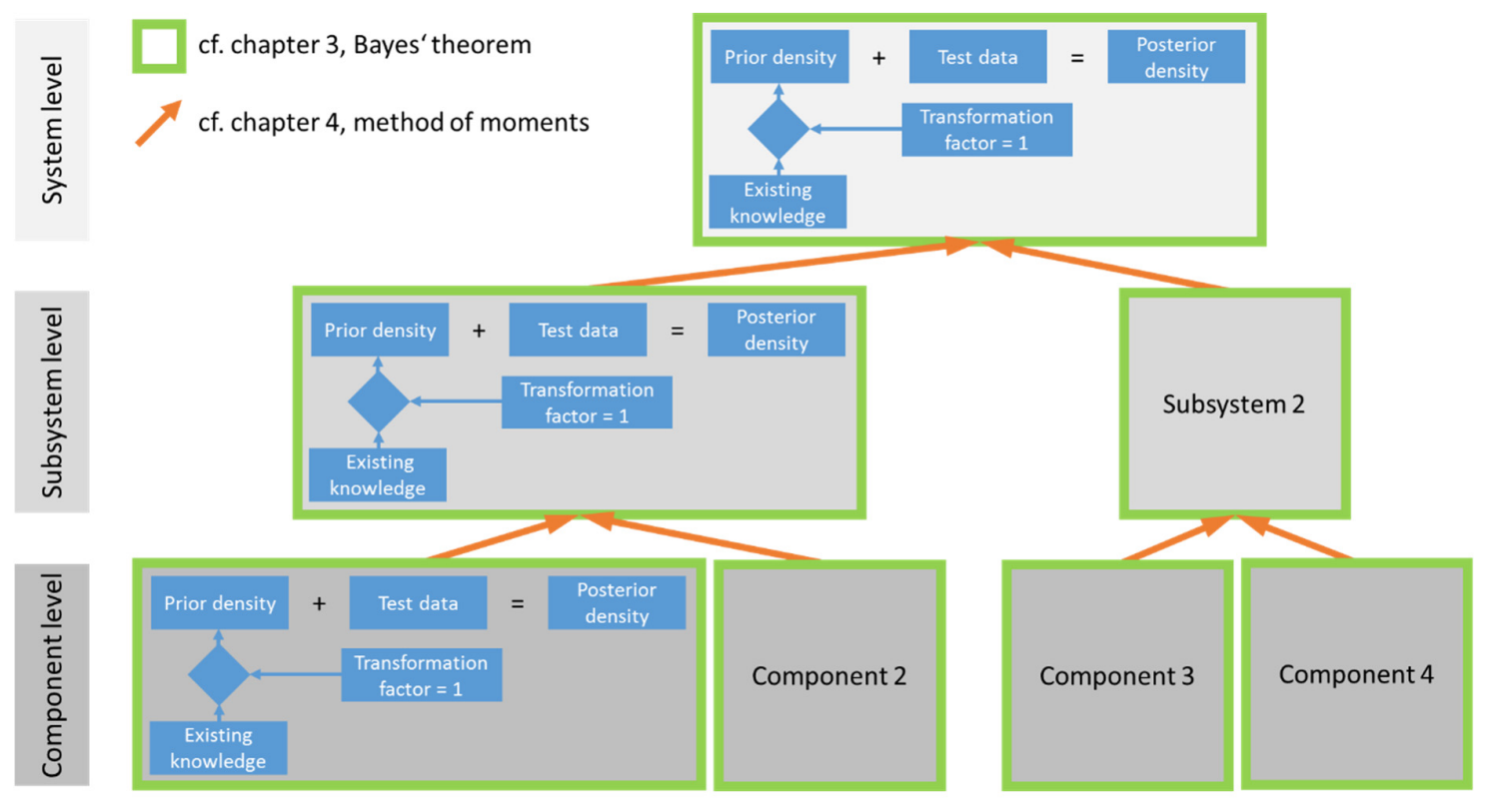

Figure 3. Approach applied to the combination of prior knowledge

\section{The EcoLife sample gearbox}

The reduced system structure which is to represent the entire EcoLife system in the context of reliability is illustrated in Figure 4 together with an image of the gearbox itself. The 15 components which are considered for the reliability prediction and validation are outlined in bold. For these, prior knowledge from calculations is included.

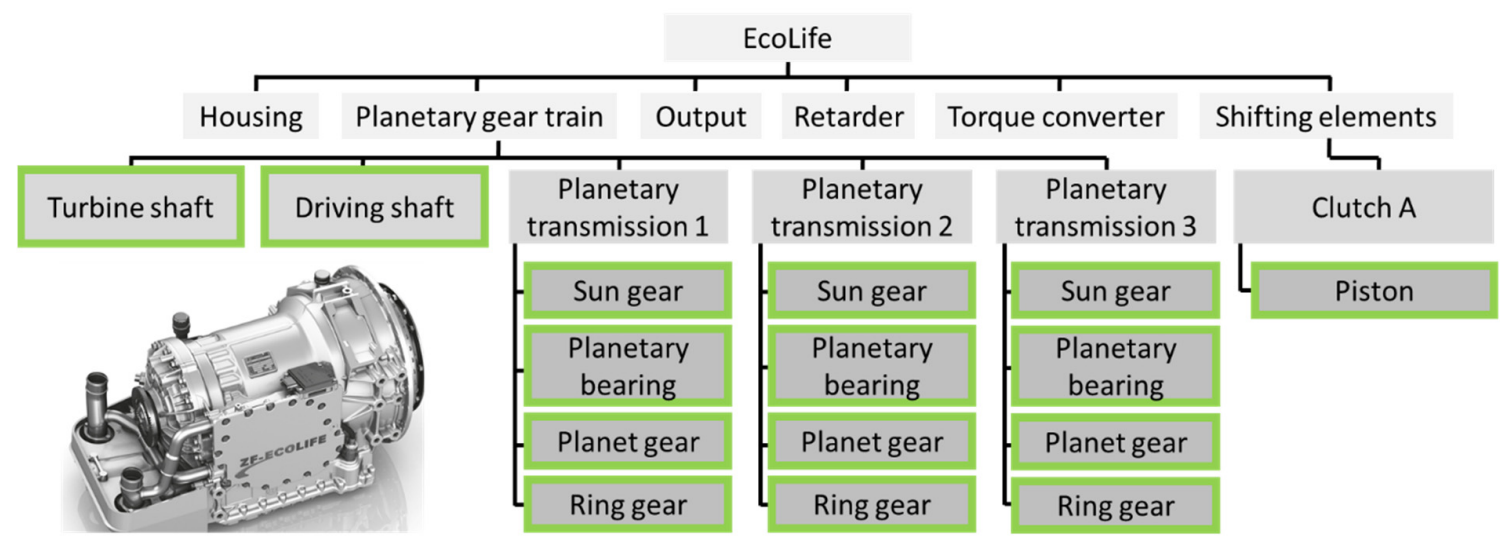

Figure 4. Simplified system structure for the EcoLife sample gearbox

Besides the simplification of the complex EcoLife into 15 components, the following assumptions are underlying:

- The system structure is strictly serial,

- The reliability densities are described by means of Beta distributions,

- The reliabilities of those components not explicitly regarded are assumed to be $\mathrm{R}\left(\mathrm{t}_{\mathrm{s}}\right)=1(100 \%)$,

- Every set of planetary gears was tested several times without failure and its components' stresses were translated into individual lifetime ratios. The independence of tests at a subsystem level (planetary transmission) and component level is provided. Test results are considered twice, i.e. both at component and subsystem level. 


\section{Calculation of the posterior reliability exemplified for planetary transmission 1}

Initially, prior knowledge from calculations for the individual components allocated to planetary gear 1 (cf. Figure 4) is combined with current test results via Bayes' theorem (cf. Chapter 3). This is exemplified for the planetary bearing component of planetary transmission 1 .

Next, each of the calculated posterior reliability densities of all 4 components (sun gear, planetary bearing, planet gear, ring gear) are combined to result in a (prior) reliability density of the superordinate system level of the planetary transmission by means of approximation using the Beta distributions' moments (cf. Chapter 4). Premises and results of this sample calculation are summarized in Table 1. Sensitive data is concealed with a hash. The variable $t_{\text {cal }}$ represents lifetime resulting from calculations.

Table 1. Premises and results from planetary bearing calculation, summary

\begin{tabular}{|l|l|l|l|}
\hline $\mathrm{t}_{\mathrm{s}}$ & $1[-]$ & $\mathrm{b}$ & $1.5[-]$ \\
\hline $\mathrm{R}_{\text {target }}\left(\mathrm{t}_{\mathrm{s}}\right)$ & $\# \%$ & $\mathrm{t}_{0}$ & $0.44[-]$ \\
\hline $\mathrm{P}_{\mathrm{A}}\left(\mathrm{R}_{\text {target }}\left(\mathrm{t}_{\mathrm{s}}\right)\right)$ & $50 \%$ & $\mathrm{~T}$ & $\#[-]$ \\
\hline $\mathrm{t}_{\text {cal }}$ & $8.72[-]$ & $\mathrm{R}_{\text {cal }}\left(\mathrm{t}_{\mathrm{s}}\right)$ & $\# \%$ \\
\hline $\mathrm{R}\left(\mathrm{t}_{\text {cal }}\right)$ & $\# \%$ & $\Delta \mathrm{n}_{\text {cal }}$ & 1.98 units \\
\hline
\end{tabular}

The reliability at the specified lifetime $R_{\text {cal }}\left(t_{s}\right)$ is calculated as follows (Bertsche and Lechner, 2004, p. 43):

$$
R_{c a l}\left(t_{s}\right)=\left(1-F\left(t_{c a l}\right)\right)^{\left(\frac{t_{s}-t_{0}}{t_{c a l}-t_{0}}\right)^{b}}
$$

The theoretical reduction of the sample size $\Delta \mathrm{n}_{\text {cal }}$ is calculated using Equation 6 .

Table 2 summarizes the test results for the component planetary bearing 1, i.e. the items of information for conditional probability, cf. Equation 1.

The value for the shape parameter $b$ of the Weibull distribution is deliberately higher. Different shape parameters $b$ result when describing empirical failure data which factually indicates a failure-free time $\mathrm{t}_{0}$ with 2- or 3-parameter Weibull distributions. The shape parameter for the 2-parameter Weibull distribution tends to be higher. As for the tests, a 2-parameter Weibull was hypothesized by ZF Friedrichshafen AG.

Table 2. Test results for planetary transmission 1, summary, 3 success runs (SR)

\begin{tabular}{|l|l|l|l|}
\hline $\mathrm{n}$ & 1 per SR & $\mathrm{L}_{\mathrm{v}} 1$ & $1.18[-]$ \\
\hline $\mathrm{b}$ & $2[-]$ & $\mathrm{L}_{\mathrm{v}} 2$ & $2.41[-]$ \\
\hline $\mathrm{r}$ & $1[-]$ & $\mathrm{L}_{\mathrm{v}} 3$ & $1.69[-]$ \\
\hline
\end{tabular}

Equations 8 are applied to calculate the descriptive parameters of the Beta distribution. The posterior reliability density functions obtained for the planetary bearing in this manner, in addition to the additional density functions of the other components in planetary transmission 1 according to the system structure (cf. Figure 4), are illustrated in Figure 5 (thin lines, dashed). Those density functions for the sun gear and the ring gear are coincidentally identical.

The reliability density of planetary transmission 1 is also illustrated (bold, dashed) in Figure 5. The descriptive parameters were calculated using Equations 8 - 10. This reliability density is regarded as a prior density function (cf. Chapter 5), as represented by the parameters $\mathrm{A}_{0}$ and $\mathrm{B}_{0}$, and is combined with the available test results. Equation 7 then becomes Equation 12.

$$
A=A_{0}+\sum_{i}\left(L v_{i} \cdot r_{i}\right)^{b_{i}} \cdot n_{i}+\Delta n_{b e r}-x ; \quad B=B_{0}+x
$$

If no calculation results are available, as is the case at the system level of the planetary transmission, this means that no prior knowledge from calculations can be used, i.e. $\Delta \mathrm{n}_{\text {cal }}=0$. 


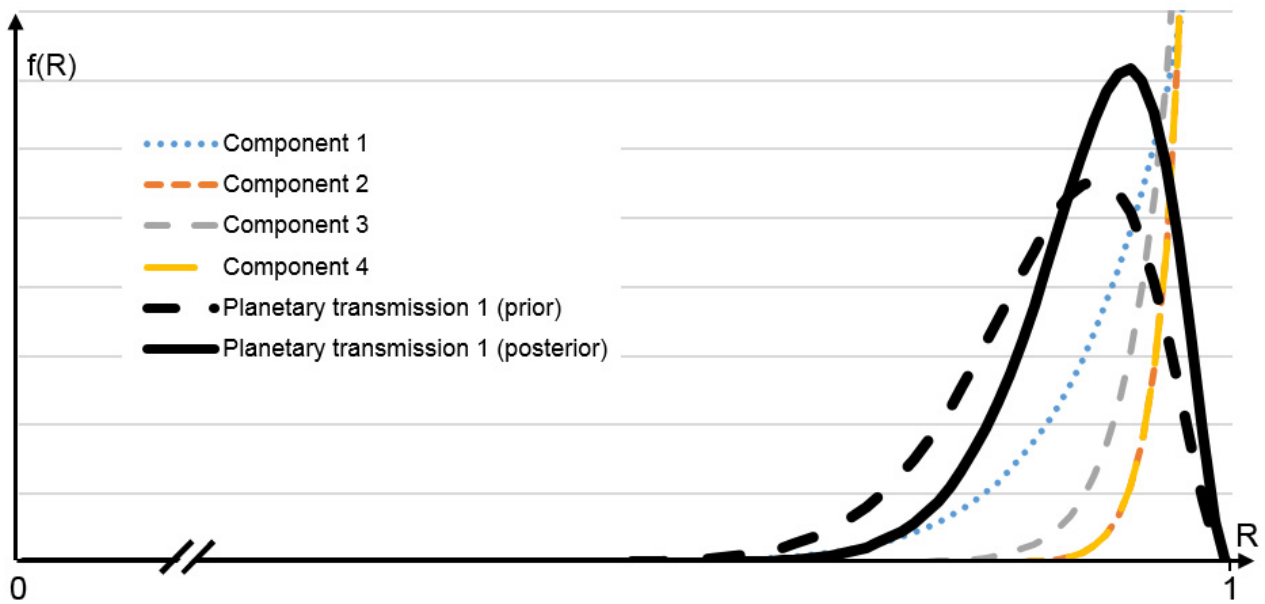

Figure 5. Reliability densities of planetary transmission 1

Planetary transmissions 1 and 3 were tested without failure in three tests with different lifetime ratios. The consideration of these test results in line with the approach described in Chapter 3 or according to Equation 12 leads to an improved (updated) statement on reliability. A certain reliability level was able to be predicted with a higher confidence level. Figure 5 also illustrates the obtained posterior density (resulting from the consideration of the test results) of planetary transmission 1 (bold, solid).

\section{Reliability densities of further components, modules and subsystems}

The reliability densities of further units according to the system structure are determined in the same way as the approach described above. Some differing aspects will be clarified below.

Turbine shaft: A uniformly distributed reliability density is considered to be prior knowledge. This corresponds mathematically with $\Delta \mathrm{n}_{\text {cal }}=1$ (cf. Krolo, 2004, p.70). Six EoL tests and one SR test at different stress levels are considered to be information with which the prior knowledge is updated. The test results are referenced to the equivalent lifetime under field conditions by means of the inverse power law. Lifetime ratios are determined. As for the Weibull shape parameter, a typical empirical value is assumed.

Driving shaft: Calculation results are combined with results from 5 SR tests. The stresses the shaft is exposed to during the different tests varies. The achieved load cycles are related to their equivalent under field stress in order to calculate lifetime ratios. The test character has no influence on the consideration of lifetime ratios. Failures are taken into account through the added "x", cf. Equation 12. By means of an amplitude modulation in accordance with (Haibach, 2006, p. 185), the different shares of the stresses are reduced to a purely alternating load.

Planetary transmissions 2 and 3: The reliability density of transmissions 2 and 3 are calculated in the same way described by way of example in Chapter 7 for that of planetary transmission 1. Again, the prior density functions stemming from calculations are considered as prior knowledge and are each combined with three EoL tests in order to obtain the posterior density functions. These posterior density functions turn into the prior density function at a superordinate system level thanks to the approximation by means of the Beta distribution.

Piston A: Calculation results concern the area identified as the most critical. In the process, different surface roughness levels were analysed. Test results are available for two different stresses (pressures) from three SR tests each. The individually bearable number of load cycles is again concluded through the inverse power law, while the exponent of the inverse power law is estimated based on similar products. The other components of the subsystem "shifting elements" are not part of the analysis of this project, which means that they are assessed with $\mathrm{R}=1$ (cf. Chapter 6). The calculated reliability density of piston $\mathrm{A}$ is therefore equal to the superordinate subsystem clutch $\mathrm{A}$, which is in turn equal to its superordinate subsystem "shifting elements".

Figure 6 illustrates inter alia the reliability densities of the subsystems "turbine shaft", "driving shaft", "planetary transmissions 1-2" and "clutch A". 


\section{System reliability density of EcoLife}

The densities of planetary transmission 1-3, together with the densities of the turbine shaft and the driving shaft, resulted in the reliability densities of the planetary gear train, cf. system structure in Figure 4. This density, combined with clutch A's density (which was said to be equal to that of shifting elements), results in the reliability density of the overall EcoLife system. The density of clutch A is described by a very high A-value, while its B-value is comparatively low (similar to the densities of the turbine shaft and the driving shaft). This implies a very high reliability. Combined with the planetary gear train, this results in the density of the overall system. The density of the planetary gear train and of the overall EcoLife system are almost identical in this model, as very high reliabilities for both the turbine shaft and the driving shaft were predicted, cf. Figure 6.

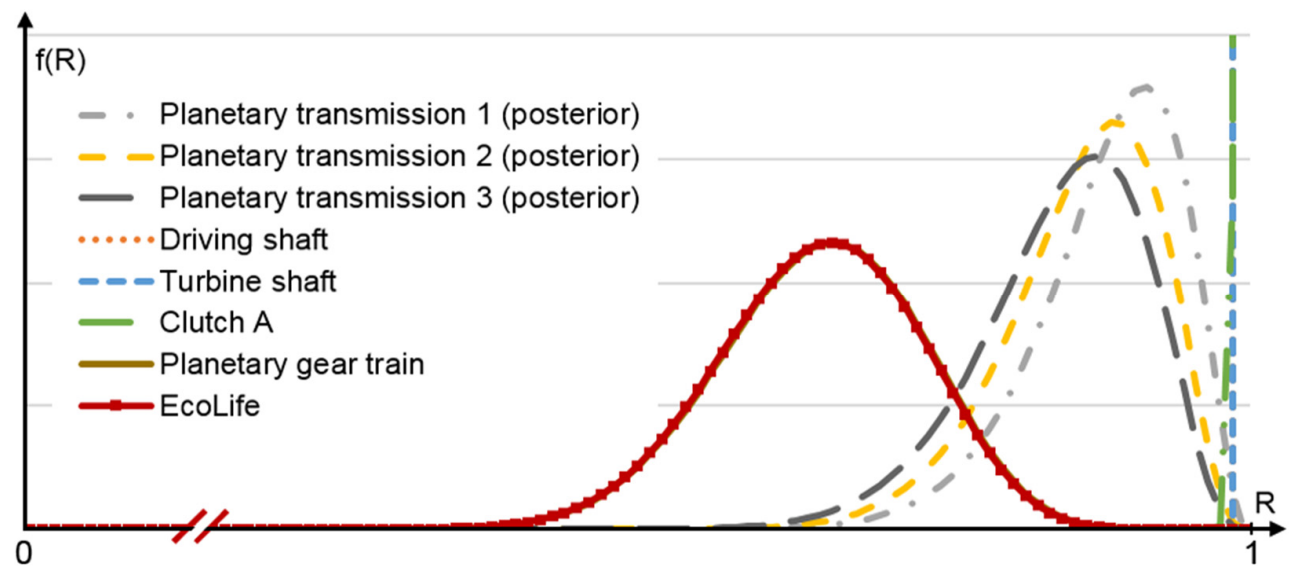

Figure 6. Reliability densities of the subsystems and the system EcoLife

\section{Validation: Comparison of predicted and observed reliability}

The accuracy of the predicted reliability is retrospectively assessed through a comparison with the reliability levels observed in the field. Documented failures form the basis for the analysis of the confidence level of the considered units. Suspensions (i.e. survivors, units without failures) were taken into account in accordance with the time-dependent production volume. Figure 7 illustrates the process applied for quantifying the number of survivors.

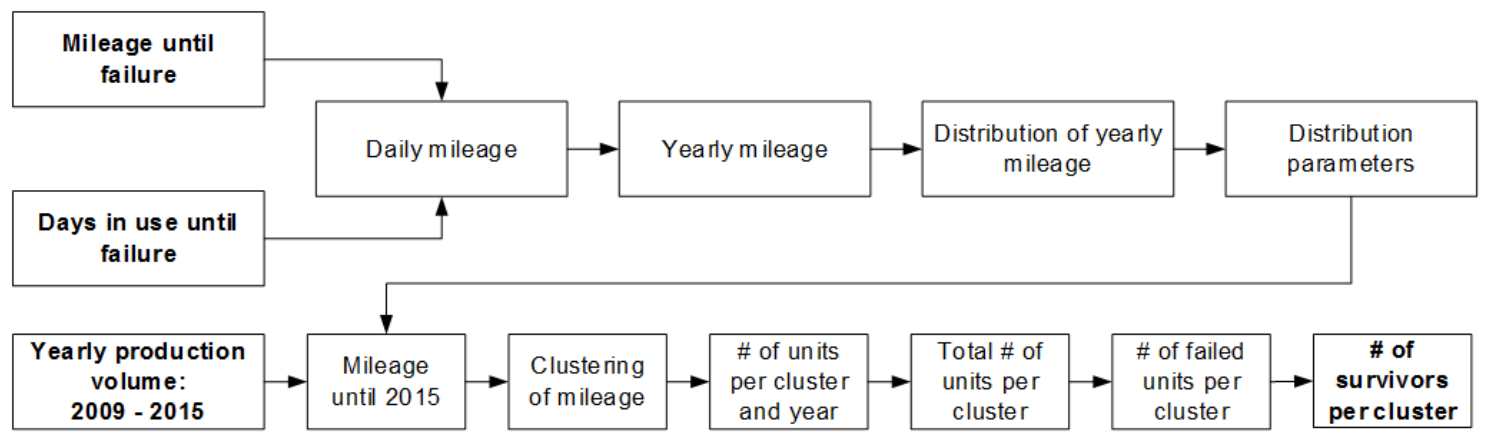

Figure 7. Approach for determining the number of survivors in field

Based on the recorded complaints, a mileage distribution was determined which serves to allocate corresponding random values to the survivors. The calculated reliability predictions $R$ at $t_{s}=1$ with a confidence level of $\mathrm{P}_{\mathrm{A}}=50 \%$ were compared to their counterpart from field data. The results of the comparison is summarized in Table 3 in terms of a reliability ratio. 
Table 3. Comparison of predicted and observed reliabilities

\begin{tabular}{|c|c|}
\hline System / Component: & Reliability ratio (observed/predicted): \\
\hline Planetary gear train & 1.92 \\
\hline Shifting elements & 0.93 \\
\hline \multicolumn{1}{|c|}{ Clutch A } & 1.00 \\
\hline \multicolumn{1}{|c|}{ Piston A } & 1.00 \\
\hline EcoLife & 1.81 \\
\hline
\end{tabular}

For piston A and clutch A, the model yields good conformity between predicted and observed reliability. In the case of the planetary gear train, the observed reliability deviates toward higher reliability levels by about half of the predicted value. This can partly be traced back to the serial system structure, where the components' low reliability levels have a severe impact on the system's reliability. The reliability of the shifting elements was slightly overestimated: One reason for this might be the high degree of simplification of the subsystem "shifting elements".

The observed reliability of the EcoLife system relates to the predicted reliability as similarly as the planetary gear train.

\section{Summary and outlook}

The method shown is capable of combining different sources of information by using Bayes' theorem and thus generating an assured statement on overall system reliability. However, the applied method seems to have a conservative tendency, even when prior knowledge is fully transferred (here, the transformation factor is $\Phi=1$, cf. Krolo, 2004, and Maisch, 2007, pp. 95-96). The presented example of reliability prediction shows that the reliability prediction is more conservative than the observed reliability, despite the integration of results from calculation as prior knowledge and the consideration of test results as a conditional probability.

The presented approach and its combination of methods is suitable for quantitative, transparent and updateable prediction of reliability at different system levels. Its fundamental operational capability and the benefit of its application have been proven. A further consideration of the transferability of the prediction to field behaviour may support the interpretation of the method-inherent conservative tendency of the prognosis, e.g. through factorial adjustments.

Results of the EoL tests entail an increasingly conservative character for the predicted posterior reliability density for method-inherent reasons. The number of failures $\mathrm{x}$ reduces Beta parameter A, but increases B (cf. Equations 8 and 12) and thus a much lower reliability results. On the contrary, if only SR tests are conducted, parameter A would not be reduced and B would remain minimal. To overcome this conservative character an alternative method for calculating Beta parameters is needed. For example, if a sufficient amount of EoL tests have been performed, a full Weibull analysis can be applied and for the resulting confidence bounds at arbitrary levels at $\mathrm{t}_{\mathrm{s}}$, beta parameters A and B can be found. The consideration of additional test results would add further accuracy to the reliability prediction. Acceleration factors ought to be integrated into the approach presented here in order to more transparently consider tests at a stress level different from the field stress. Transformation factors would only realize a partial - yet still scalable - transfer of prior knowledge, applicable in cases where the prior knowledge stems from former product generations for which comparability is limited, for example. The effect of alternative assumptions on the kind of reliability distribution from calculations ought to be analysed.

The demonstrated structure of the approach for predicting reliability at different system levels by means of combining several different subsystem reliabilities of diverse origin (here: calculation and test) is practicable and suitable for a repetitive update of the reliability statement $\left(\mathrm{R}, \mathrm{P}_{\mathrm{A}}\right)$. For this purpose, the obtained posterior density needs to be understood as the new prior density, which is then combined with the up-to-date results from tests or calculations. As a consequence, the accuracy of the reliability statement increases. 


\section{References}

Bayes, T. and Price, R. (1763), "An Essay towards Solving a Problem in the Doctrine of Chances. By the Late Rev. Mr. Bayes, F. R. S. Communicated by Mr. Price, in a Letter to John Canton, A. M. F. R. S”, Philosophical Transactions of the Royal Society of London, Vol. 53, pp. 370-418. https://doi.org/10.1098/rstl.1763.0053

Bertsche, B. and Lechner, G. (2004), Zuverlässigkeit im Fahrzeug- und Maschinenbau: Ermittlung von Bauteilund System-Zuverlässigkeiten, VDI-Buch, 3rd ed., Springer, Berlin. https://doi.org/10.1007/3-540-34996-0

Haibach, E. (2006), Betriebsfestigkeit: Verfahren und Daten zur Bauteilberechnung, VDI-Buch, Springer, Berlin, Heidelberg. https://doi.org/10.1007/3-540-29364-7

Krolo, A. (2004), Planung von Zuverlässigkeitstests mit weitreichender Berücksichtigung von Vorkenntnissen, $\mathrm{PhD}$ thesis, Institut für Maschinenelemente, Universität Stuttgart, Stuttgart. http://doi.org/10.18419/opus-4023

Krolo, A. and Bertsche, B. (2003), "An approach for the advanced planning of a reliability demonstration test based on a Bayes procedure", Proceedings of the Annual Reliability and Maintainability Symposium, Tampa, Florida, USA, January 27-30, 2003, IEEE, pp. 288-294. https://doi.org/10.1109/RAMS.2003.1181940

Maisch, M. (2007), Zuverlässigkeitsorientiertes Erprobungskonzept für Nutzfahrzeuggetriebe unter Berücksichtigung von Betriebsdaten, PhD thesis, Universität Stuttgart, Stuttgart. http://doi.org/10.18419/opus4119

Martz, H.F., Waller, R.A. and Fickas, E.T. (1988), "Bayesian Reliability Analysis of Series Systems of Binomial Subsystems and Components", Technometrics, Vol. 30 No. 2, pp. 143-154. https://doi.org/10.2307/1270159

Thompson, W.E. and Haynes, R.D. (1980), "On the reliability, availability and bayes confidence intervals for multicomponent systems", Naval Research Logistics Quarterly, Vol. 27 No.3, pp. 345-358. https://doi.org/10.1002/nav.3800270302

Zeiler, P. and Bertsche, B. (2015), "Component reliability allocation and demonstration test planning based on system reliability confidence limit”, In: Podofillini, L., Sudret, B., Stojadinovic, B., Zio, E. and Kröger, W. (Eds.), Safety and Reliability of Complex Engineered Systems, CRC Press, London, pp. 1805-1811. https://doi.org/10.1201/b19094-234

Alexander Grundler, Research Assistant

University of Stuttgart, Institute of Machine Components

Pfaffenwaldring 9, 70569 Stuttgart, Germany

Email: Alexander.Grundler@ima.uni-stuttgart.de 